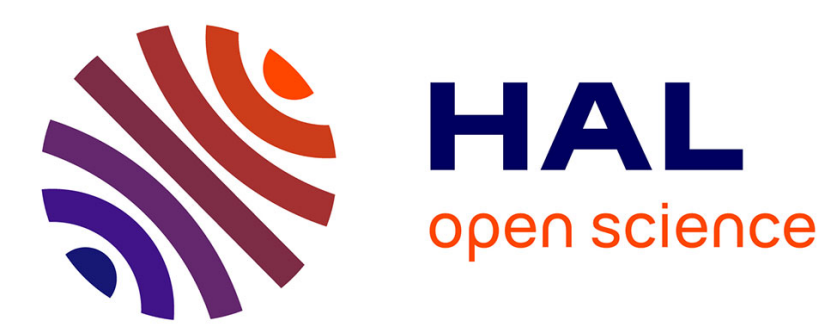

\title{
Effect of tube heat conduction on the single branch pulsating heat pipe start-up
}

\author{
Vadim Nikolayev
}

\section{To cite this version:}

Vadim Nikolayev. Effect of tube heat conduction on the single branch pulsating heat pipe start-up. International Journal of Heat and Mass Transfer, 2016, 95, pp.477. 10.1016/j.ijheatmasstransfer.2015.12.016 . cea-01367172

\section{HAL Id: cea-01367172 https://hal-cea.archives-ouvertes.fr/cea-01367172}

Submitted on 15 Sep 2016

HAL is a multi-disciplinary open access archive for the deposit and dissemination of scientific research documents, whether they are published or not. The documents may come from teaching and research institutions in France or abroad, or from public or private research centers.
L'archive ouverte pluridisciplinaire HAL, est destinée au dépôt et à la diffusion de documents scientifiques de niveau recherche, publiés ou non, émanant des établissements d'enseignement et de recherche français ou étrangers, des laboratoires publics ou privés. 


\title{
Effect of tube heat conduction on the single branch pulsating heat pipe start-up
}

\author{
Vadim S. Nikolayev \\ Service de Physique de l'Etat Condensé, CEA, CNRS, Université Paris-Saclay, CEA Saclay, 91191 Gif-sur-Yvette Cedex, France
}

\section{A R T I C L E I N F O}

\section{Article history:}

Received 2 October 2015

Received in revised form 4 December 2015

Accepted 10 December 2015

Available online 29 December 2015

\section{Keywords:}

Evaporation

Gas-liquid meniscus

Oscillations

Instability

Pulsating heat pipe

PHP

OHP

Start-up

\begin{abstract}
A B S T R A C T
The oscillation inception in the single-branch pulsating heat pipe (PHP, called also oscillating heat pipe) has been studied in the presence of the heat conduction along the PHP tube, with the imposed both evaporator heat power and condenser temperature. A start-up regime caused rather by the meniscus/film evaporation than boiling has been considered. The dynamic equilibrium system state has been analyzed, where the liquid film is absent and the meniscus is located at a position where the tube temperature corresponds to the saturation temperature. The temporal evolution of the system responding to an initial fluctuation shows a non-linear response even for small fluctuations. The stability of the equilibrium state has been analyzed. The stability threshold corresponds to the start-up criterion. The main result of the above analysis is the independence of the start-up criterion of the liquid film properties (film shape and thickness). This result applies to the multi-branch PHP too. Due to the large tube thermal inertia, influence of the temporal variation of the tube temperature on the threshold can be neglected; only the equilibrium spatial temperature distribution along the tube matters. The start-up threshold value is determined for the temperature gradient along the tube, more specifically, its equilibrium value at the equilibrium meniscus location. It depends only weakly on other system parameters like condenser temperature or adiabatic section length. An analytical expression for the threshold has been obtained. The start-up power scales like square root of the tube heat conductivity. The liquid viscous dissipation is found to be much less important than the energy dissipation via the fluid and solid heat transfer.
\end{abstract}

(c) 2015 Elsevier Ltd. All rights reserved.

\section{Introduction}

The pulsating (or oscillating) heat pipe (PHP) is a looped capillary tube that meanders between hot and cold spots that form evaporator and condenser sections, respectively. The tube is filled with a pure fluid in such a way that liquid plugs and gas bubbles coexist inside. When the temperature difference between the evaporator and condenser exceeds a threshold, the self-sustained oscillations of the plugs and bubbles appear. The PHP is extremely attractive for various industrial applications because of high thermal performance and manufacturing simplicity. However the PHP functioning is not completely understood; the absence of predictive tools that would allow their dimensioning is a substantial obstacle to their development. The reason for that is, on one hand, a multitude of complicated physical phenomena involved into their functioning [1,2], and on the other, its intrinsic non-stationarity. Because of the PHP complexity, their onedimensional (1D) modeling has been applied initially [3] where only liquid and gas plugs with dry tube walls were modeled. The

E-mail address: vadim.nikolayev@cea.fr model has been extended later [4] to account for the liquid films (on the internal tube walls) through which most of the heat and mass exchange occurs.

The crucial question about the PHPs concerns their start-up, i.e. the oscillation inception. One can approach this question by direct simulation [4]. Because of the multitude of parameters that influence the start-up and different start-up modes [5], direct simulation of the multi-branch PHP is not the best way to study the start-up criteria. To gain understanding of the PHP start-up, one needs to begin with simple PHP geometries. In this article we consider the start-up of the simplest, single branch PHP (Fig. 1) for which some analytical results are possible to obtain [6,7]. It is a straight capillary with a sealed end, which is heated (evaporator). The gas bubble is confined between the sealed end and a liquid plug. The condenser end of the capillary is connected to a large reservoir filled partially with the liquid at constant pressure $p_{r}$.

One can distinguish two main start-up modes: via boiling inside liquid plugs situating in evaporator or via evaporation from liquid menisci $[5,8]$. The first regime is often associated with a strong evaporator temperature overshoot that appears between the power switching-on and the oscillation beginning after which the 


\section{Nomenclature}

$D \quad$ thermal diffusion coefficient $\left[\mathrm{m}^{2} / \mathrm{s}\right]$

$d \quad$ tube diameter [m]

$e \quad$ dimensionless volume element length, base of the natural logarithm

$f \quad$ dimensionless viscous friction

$h_{l g} \quad$ latent heat $[\mathrm{J} / \mathrm{kg}]$

$j \quad$ volume heat supply $\left[\mathrm{W} / \mathrm{m}^{3}\right]$

$k \quad$ heat conductivity $[\mathrm{W} /(\mathrm{m} \cdot \mathrm{K})]$

$L \quad$ length [m]

$m \quad$ mass $[\mathrm{kg}]$

$N \quad$ number of volume elements

$P \quad$ heat power [W]

$p$ pressure [Pa]

$q \quad$ heat flux $\left[\mathrm{W} / \mathrm{m}^{2}\right]$

$R \quad$ gas constant for gas $[\mathrm{J} /(\mathrm{kg} \cdot \mathrm{K})]$

$r \quad$ dimensionless velocity amplitude

$S \quad$ cross-section area $\left[\mathrm{m}^{2}\right]$

$T$ temperature [K]

$t$ time [s]

$U \quad$ heat transfer coefficient $\left[\mathrm{W} /\left(\mathrm{K} \cdot \mathrm{m}^{2}\right)\right]$

$V \quad$ meniscus velocity $[\mathrm{m} / \mathrm{s}]$

$x \quad$ abscissa $[\mathrm{m}]$

Greek symbols

$\begin{array}{ll}\alpha & \text { dimensionless evaporator length } \\ \beta & \text { dimensionless heating power } \\ \chi, \xi, \zeta & \text { dimensionless constants defined in Eq. (25) } \\ \delta & \text { liquid film thickness [m] } \\ \eta & \text { reduced condenser temperature }\end{array}$

$\gamma \quad$ adiabatic index

$\lambda$ dimensionless coordinate

$v \quad$ liquid kinematic viscosity $\left[\mathrm{m}^{2} / \mathrm{s}\right]$

$\Omega \quad$ dimensionless parameter characterizing the tube thermal inertia

$\Pi \quad$ oscillation period [s]

$\psi \quad$ dimensionless gas temperature

$\rho \quad$ density $\left[\mathrm{kg} / \mathrm{m}^{3}\right]$

$\sigma \quad$ dimensionless saturation curve slope

$\tau \quad$ dimensionless time

$\theta, \mu, \phi \quad$ dimensionless constants defined in Eq. (36)

$\varepsilon \quad$ reduced adiabatic length

$\varphi, \kappa \quad$ dimensionless phase shifts

\section{Subscripts}

c condenser

e evaporator

$f \quad$ film

$g \quad$ gas

$l \quad$ liquid

m meniscus

$o \quad$ outer tube wall

$r \quad$ reservoir

$s \quad$ solid tube wall material, internal tube wall

sat saturation

sens sensible

$t \quad$ total

$V \quad$ at constant volume and for gas phase

$0 \quad$ at $t=0$ temperature drops. It is related to the energy barrier required for the bubble nucleation. The second regime exhibits a smaller temperature overshoot (if any) and for this reason is more advantageous because it provides a better temperature stability. For this reason, in this article we study this latter regime that we call "soft" to distinguish from the "hard" regime with a bigger temperature overshoot.

The "film evaporation-condensation" (FEC) model introduced in [6] is used here. It is a 1D model. The heat/mass exchange is controlled mainly by the thermal conduction in the liquid films described by the terms $\propto\left(T_{s}-T_{\text {sat }}\right)$, where $T_{\text {sat }}$ is calculated for the current gas pressure $p$ and $T_{S}$ is the temperature of the internal tube wall. The FEC model describes large amplitude oscillations during which the meniscus sweeps both the condenser and the evaporator. The FEC model agrees quantitatively with the experimental results [6] on the single branch PHP. Recently, the FEC model has been validated against the data obtained with another experimental set-up [9]. It described most features observed

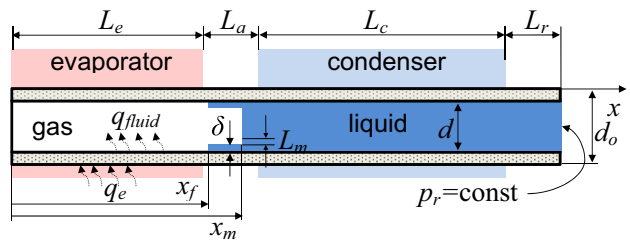

Fig. 1. Single branch PHP within the lumped meniscus approximation. The total tube length $L_{t}=L_{e}+L_{c}+L_{r}$ includes an effective length $L_{r}$ representing an amount of the liquid in the reservoir that takes part in the oscillating motion; $L_{e}$ and $L_{c}$ are the lengths of the respective tube sections. experimentally (like the intermittency of oscillations observed by both $[6,9])$.

In the preceding article [7], the start-up has been studied for the simplest thermal boundary conditions: the imposed temperature at the internal tube walls both in the condenser and the evaporator. The temperature varied stepwise along the tube (from condenser to evaporator) for the case of PHP without adiabatic section. In the present article, we consider a more realistic case of the smooth temperature variation in the presence of adiabatic section. The thermal boundary conditions approach the experimental situation. While the condenser temperature $T_{c}$ is imposed at the internal tube walls, the thermal conduction along the tube is introduced so that the tube temperature is allowed to vary both spatially and temporally. A constant evaporator heat power $P_{e}$ is imposed.

When experimentalists consider the PHP start-up, they speak usually of what happens after the power switching-on. Two situations are then possible: either PHP begins to oscillate or it comes to some stationary non-oscillating state of dynamic equilibrium. One does not need to consider the switching-on procedure when approaching the start-up problem theoretically. One needs to find instead an equilibrium state and see if it is stable or unstable with respect to a small fluctuation. The stable state corresponds to that found experimentally and means the absence of oscillation while the instability corresponds to the oscillation start-up.

The article is structured as follows. The model is summarized in Section 2. The equilibrium state for such a system is identified in Section 3. The stability of the equilibrium state is studied in Section 4. The oscillation threshold (i.e. start-up criterion) is analyzed in Section 4.3. The results are summarized in Section 5. 


\section{Film evaporation-condensation model in the presence of the tube thermal conduction}

The thermal diffusion inside the solid material of the tube is introduced similarly to [10] where the effect of tube conduction has been first introduced with the application to PHPs:

$\frac{\partial T_{s}}{\partial t}=D_{s} \frac{\partial^{2} T_{s}}{\partial x^{2}}+\frac{j_{s}}{\rho_{s} c_{s}}$

It is assumed that the tube properties do not vary along the tube. All other variables related to the heat diffusion depend both on $x$ and $t$. The thermal boundary conditions mimic the cryogenic experiment [11]: the external tube surface in the evaporator and adiabatic sections is assumed to be thermally isolated from the environment. The heat is supplied to the tube through its inner and outer walls. The equivalent 1D volume heat supply to the fluid is obtained by multiplication of the heat flux by the element of the respective wall area and division by the tube material volume element;

$j_{s}=\frac{\pi}{S_{s}} \begin{cases}q_{e} d_{o}-q_{\text {fluid }} d & \text { if } 0<x \leqslant L_{e}, \\ -q_{\text {fluid }} d & \text { if } L_{e}<x \leqslant L_{e}+L_{a},\end{cases}$

where $S_{s}=\pi\left(d_{o}^{2}-d^{2}\right) / 4$ is the tube material cross-section area. The heat flux $q_{e}$ applied to the evaporator is distributed uniformly and is thus constant

$q_{e}=\frac{P_{e}}{\pi d_{0} L_{e}}$.

The heat flux $q_{\text {fluid }}=U_{\text {fluid }}(x)\left(T_{s}-T_{\text {fluid }}\right)$ is transferred from the internal tube wall to the fluid. It can vary in time and space. The significance of $T_{\text {fluid }}$ is

$T_{\text {fluid }}= \begin{cases}T & \text { if } x \in \text { gas bubble, } \\ T_{\text {sat }} & \text { if } x \in \text { liquid film, } \\ T_{l} & \text { if } x \in \text { bulk liquid }\end{cases}$

and heat exchange coefficient $U_{\text {fluid }}$ is either $U_{g}, U_{f}$ or $U_{l}$ for the respective regions. All three heat transfer coefficients are assumed to be constant.

The thermal diffusion in the liquid is described by a similar equation

$\frac{\partial T_{l}}{\partial t}=D_{l} \frac{\partial^{2} T_{l}}{\partial x^{2}}+\frac{\pi d U_{l}}{\rho_{l} c_{l} S}\left(T_{s}-T_{l}\right)$,

where $S=\pi d^{2} / 4$.

The choice of the boundary conditions for the thermal diffusion problem is of importance. It is assumed here that the condenser is efficient enough to impose the constant temperature at its boundary,

$T_{s}\left(x=L_{e}+L_{a}\right)=T_{c}$

The adiabatic condition is chosen at the sealed end of the tube,

$\left.\frac{\partial T_{s}}{\partial x}\right|_{x=0}=0$.

The thickness of thermal boundary layer formed near the liquid

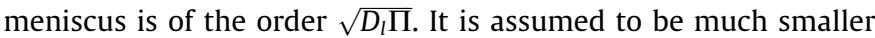
than the liquid penetration length into the adiabatic section. We will see below that such an assumption is justified. This means that one may choose

$T_{l}\left(x=L_{e}+L_{a}\right)=T_{c}$.

With these assumptions, the integration interval for Eqs. (1) and (5) may be limited to $0<x<L_{\rho}+L_{a}$ and to $x_{m}<x<L_{\rho}+L_{a}$, respectively. The saturation temperature at the liquid meniscus
$T_{l}\left(x=x_{m}\right)=T_{\text {sat }}$

provides the remaining boundary condition.

The gas mass exchange is obtained from the energy balance at the gas-liquid interface that states that the heat flux from the liquid side is spent for vaporization (or condensation if negative). The total vaporization rate

$\dot{m}=\dot{m}_{f}+\dot{m}_{m}$,

where dot means the time derivative, is obtained by integration of the flux over the gas-liquid interface. The latter is divided into two parts. The first is the flat film surface and the corresponding contribution

$\dot{m}_{f}=\frac{U_{f} \pi d}{h_{l g}} \int_{x_{f}}^{x_{m}}\left[T_{s}(x)-T_{s a t}\right] \mathrm{d} x$.

is non-zero if the film exists. The second part of the gas-liquid interface on which the mass exchange occurs is the meniscus, more precisely, its small part $L_{m}$ (Fig. 1) on which the heat flux is non-negligible [2]. This meniscus part is adjacent to the film. The corresponding contribution is

$\dot{m}_{m}=\frac{U_{m} L_{m} \pi d}{h_{l g}}\left[T_{s}\left(x_{m}\right)-T_{s a t}\right]$.

According to the film evaporation-condensation model $[6,4,7]$, the film edge dynamics is defined only by the non-negative evaporation part $\dot{m}_{f, e}$ of $\dot{m}_{f}$,

$\dot{x}_{f}= \begin{cases}V & \text { if } x_{f} \geqslant x_{m}, \quad V<0 \\ \dot{m}_{f, e} /\left(\rho_{l} \pi d \delta\right) & \text { otherwise }\end{cases}$

where

$\dot{x}_{m}=V$.

The first line of Eq. (10) corresponds to the meniscus advancing over the dry evaporator (where $x_{f}=x_{m}$ ). In principle, $x_{f} \leqslant x_{m}$ so that the condition $x_{f}>x_{m}$ may seem to be unnecessary. It is however useful when the solution of Eq. (10) becomes slightly larger than $x_{m}$ both in a numerical calculation (because of the finiteness of the time step) and in the approximate analytical approach considered in the Appendix B. The second line of Eq. (10) corresponds to the Landau-Levich film deposition characteristic to the Taylor bubble motion $[2,12]$ and its evaporation. Such an expression assumes a constant film thickness $\delta$. It will be shown in the following that the film parameters (shape, thickness) have no incidence on the PHP start-up.

According to Eq. (8), evaporation occurs on the film portion corresponding to $x_{f}<x<x_{\text {sat }}$, where $x_{\text {sat }}$ is defined by the condition $T_{s}\left(x=x_{\text {sat }}\right)=T_{\text {sat }}$. The film evaporation rate is then

$\dot{m}_{f, e}=\frac{U_{f} \pi d}{h_{l g}} \int_{x_{f}}^{x_{s a t}}\left[T_{s}(x)-T_{s a t}\right] \mathrm{d} x$.

Note that such a formulation provides the film edge pinning at the point $x_{f}=x_{\text {sat }}$ during the film evaporation: if a small positive "overshoot" $x_{f}-x_{\text {sat }}$ occurs due to a numerical error, the backwards edge motion is induced due to the second line of Eq. (10).

A thermal boundary layer is allowed to exist in the gas, so that the temperature $T$ of its bulk may be different from that of the gas-liquid interface (which is at $T_{\text {sat }}(p)$ ). This is possible due to the smallness of heat diffusion in the gas [6]. The experiment [11] shows that the gas temperature is several degrees higher than $T_{\text {sat }}(p)$ so that the gas evolves outside the saturation curve. For this reason its equation of state can be approximated with a good precision by the ideal gas equation

$p=\frac{m R T}{S x_{m}}$, 
where $m$ is the gas mass. The gas energy balance [13] reads

$m c_{V} \dot{T}=\dot{m} R T+P_{\text {sens }}-p S V$.

The sensible heat exchange of the tube with the gas in the evaporator is accounted for by the term

$P_{\text {sens }}=U_{g} \pi d \int_{0}^{x_{f}}\left[T_{s}(x)-T\right] \mathrm{d} x$.

The heat exchange coefficient $U_{g}$ is proportional to the gas heat conductivity $k_{g}$. Because of its smallness, the sensible heat exchange with the gas is much weaker than the exchange by evaporation/condensation. Its impact on the oscillations will be discussed below. The momentum equation for the liquid plug reads

$m_{l} \dot{V}=\left(p-p_{r}\right) S-8 \pi v \rho_{l}\left(L_{t}-x_{m}\right) V$,

where

$m_{l}=\rho_{l}\left(L_{t}-x_{m}\right) S$

is the liquid mass. The last term of Eq. (16) is the viscous friction force corresponding to the Poiseuille liquid flow which is the small velocity term of a more general expression [6]. The small velocity limit is always applicable at the startup moment. Note that since the liquid plug is usually long, dissipation in the single branch PHP is dominated by the flow far from the meniscus where the Poiseuille flow occurs. This is contrary to the multibranch PHP case where the dissipation is defined by the recirculation flow caused by the front and rear menisci [1].

Five governing ordinary differential Eqs. (7), (10), (11), (14), and (16) of the model need to be solved together with the heat diffusion problems $(1,5)$ with the boundary conditions $(6)$ to find the temporal evolution of the PHP.

\section{Equilibrium state}

First one needs to identify the equilibrium state (denoted by the over bar hereafter), the stability of which will be analyzed next. Strictly speaking, since the heat is supplied and dissipated it is the dynamic equilibrium rather than truly equilibrium state. However we will call it "equilibrium" for the sake of brevity.

\subsection{Equilibrium conditions}

The equilibrium conditions can be found by putting to zero all the time derivatives. One deduces from Eqs. (8) and (9) that the only way to cancel $\dot{m}_{f}$ and $\dot{m}_{m}$ simultaneously is to choose

$\bar{x}_{f}=\bar{x}_{m}=\bar{x}_{s a t}$,

where $\bar{x}_{\text {sat }}$ is defined via the equation

$\bar{T}_{s}\left(x=\bar{x}_{\text {sat }}\right)=\bar{T}_{\text {sat }} \equiv T_{\text {sat }}(\bar{p})$.

This means that the meniscus situates at a point where the internal wall temperature is equal to that of saturation. Such a point cannot situate in the condenser because in the opposite case only the trivial equilibrium solution $T_{s}(x)=T_{c}$ exists only for $P_{e}=0$, so that

$0<\bar{\chi}_{\text {sat }}<L_{e}+L_{a}$.

Eqs. (11) and (16) yield

$\bar{V}=0, \quad \bar{p}=p_{r}$

and Eq. (10) is satisfied automatically. From Eq. (13) one gets

$\bar{m}=\frac{p_{r} S \bar{x}_{s a t}}{R \bar{T}}$.

The equilibrium liquid mass is $\bar{m}_{l}=\rho_{l} S\left(L_{t}-\bar{x}_{s a t}\right)$.

Eq. (14) requires that $\bar{P}_{\text {sens }}=0$,

$\int_{0}^{\bar{x}_{s a t}}\left[\bar{T}_{s}(x)-\bar{T}\right] \mathrm{d} x=0$.

that should hold for any equilibrium state.

\subsection{Dimensionless formulation}

The characteristic length $L=\sqrt{S_{s} k_{s} /\left(\pi d U_{g}\right)}$ of the heat exchange in the dry tube portion is chosen to make the lengths dimensionless. All temperatures are made dimensionless with $\bar{T}_{\text {sat }}$. The following dimensionless parameters are introduced:

$\lambda=x / L$

$\alpha=L_{e} / L$

$\varepsilon=L_{a} / L_{e}$

$\chi=\bar{\chi}_{\text {sat }} / L_{e}$,

$\eta=\left(\bar{T}_{\text {sat }}-T_{c}\right) / \bar{T}_{\text {sat }}$,

$\psi=\bar{T} / \bar{T}_{\text {sat }}$,

$\beta=P_{e} /\left(\bar{T}_{s a t} L_{e} \pi d U_{g}\right)$,

$\xi=U_{l} / U_{g}$

$\zeta=k_{S} S_{s} /\left(k_{l} S\right)$.

The equations for dimensionless equilibrium temperatures denoted with the hats read

$\hat{T}_{s}^{\prime \prime}+\left\{\begin{array}{ll}\psi-\hat{T}_{s}, & 0 \leqslant \lambda<\lambda_{m} \\ \xi\left(\hat{T}_{l}-\hat{T}_{s}\right), & \text { otherwise }\end{array}\right\}=\left\{\begin{array}{ll}-\beta, & 0 \leqslant \lambda<\alpha \\ 0, & \text { otherwise }\end{array}\right\}$,

$\hat{T}_{l}^{\prime \prime}+\xi \zeta\left(\hat{T}_{s}-\hat{T}_{l}\right)=0$,

where the primes mean the derivatives over $\lambda$. The problem for $\hat{T}_{s}$ is solved for $\lambda \in[0, \alpha(1+\varepsilon)]$, while that for $\hat{T}_{l}$ is solved for $\lambda \in\left[\lambda_{m}, \alpha(1+\varepsilon)\right]$. Note that $\lambda_{m}=\bar{\chi}_{\text {sat }} \equiv \alpha \chi$ is the dimensionless equilibrium meniscus position, while $\alpha(1+\varepsilon)$ is the dimensionless $L_{e}+L_{a}$ value. The boundary conditions (6) yield to

$\hat{T}_{s}^{\prime}(0)=0$,

$\hat{T}_{s}(\alpha(1+\varepsilon))=\hat{T}_{l}(\alpha(1+\varepsilon))=1-\eta$,

$\hat{T}_{l}\left(\lambda_{m}\right)=1$

Eq. (26a) suggests two cases to be considered separately: (A) $1 \leqslant \chi<1+\varepsilon$ (meniscus located in adiabatic section at equilibrium) and (B) $0 \leqslant \chi<1$ (meniscus located at evaporator). Consider first the case $A$. It is the most commonly encountered case. It admits an analytical solution,

$\hat{T}_{s}= \begin{cases}\psi+\beta+C_{e} \cosh (\lambda), & \text { if } 0 \leqslant \lambda<\alpha, \\ \psi+C_{a}^{1} e^{\lambda}+C_{a}^{2} e^{-\lambda}, & \text { if } \alpha \leqslant \lambda<\lambda_{m}, \\ 1+\eta \frac{\chi-\lambda / \alpha}{1+\varepsilon-\chi}, & \text { if } \lambda_{m} \leqslant \lambda<\alpha(1+\varepsilon),\end{cases}$

where

$C_{e}=\frac{1-\psi-\beta \cosh [\alpha(\chi-1)]}{\cosh (\alpha \chi)}$,

$C_{a}^{1}=\frac{1-\psi-\beta \sinh (\alpha) e^{-\alpha \chi}}{2 \cosh (\alpha \chi)}$,

$C_{a}^{2}=\frac{1-\psi+\beta \sinh (\alpha) e^{\alpha \chi}}{2 \cosh (\alpha \chi)}$,

$\psi=1+\beta \frac{\alpha \cosh (\alpha \chi)-\sinh (\alpha)}{\sinh (\alpha \chi)}$,

$\chi=\varepsilon+1-\frac{\eta}{\beta \alpha^{2}}$ 
are obtained from Eqs. (27) and (28), the conditions of continuity of $\hat{T}_{s}(\lambda)$ and $\hat{T}_{s}^{\prime}(\lambda)$ at $\lambda=\lambda_{m}$, and the dimensionless counterpart of Eq. (24). Since $\hat{T}_{s}$ changes linearly along the liquid-covered portion of the solid, Eq. (26a) requires that

$$
\hat{T}_{l}(\lambda)=\hat{T}_{s}(\lambda) \text { for } \lambda \geqslant \lambda_{m}
$$

Because the last condition means the absence of the solidliquid heat transfer, the solution is independent of the liquid properties, i.e. of the parameters $\xi, \zeta$; the temperature distribution in both liquid and solid is linear, cf. the last line of Eq. (29). Because of the constraint $\chi \leqslant 1+\varepsilon$, Eq. (30e) implies $\eta>0$ or $\bar{T}_{s a t}>T_{c}$ in the dimensional variables. The constraint $\chi>1$ shows also that the case A corresponds to the criterion

$\beta>\frac{\eta}{\alpha^{2} \varepsilon}$,

i.e., to evaporator power larger than a threshold.

The behavior related to a change of the condenser temperature, i.e., the reduced parameter $\eta$ that enters the stability analysis through the parameter $\chi$ only seems to be counter-intuitive. One usually discusses the PHP startup in terms of difference between the evaporator and condenser temperatures so that a decrease of $T_{c}$ is expected to lead to the same result as an increase of $P_{e}$. The actual tendency is however opposite. While an increase of $P_{e}$ (i.e., of $\beta$ ) causes an increase of $\chi$, a decrease of $T_{c}$ (increase of $\eta$ ) causes a decrease of $\chi$, cf. Eq. (30e). This situation is caused by the fact that the pressure imposes the meniscus temperature at equilibrium and thus becomes an important parameter defining the temperature distribution along the PHP and thus the startup conditions. The same feature should hold for the multi-branch PHP with an open end.

An example for the equilibrium temperature distribution $T_{s}(x)$ along the tube is shown in Fig. 2. Note that Eq. (31) holds for the case of Fig. 2 because both curves correspond to the case A.

In the opposite case B (i.e. when the evaporator power is small) one has $\chi<1$. This case is rarely encountered in practice because of larger startup threshold, see below. The solution for this case is more complicated because it depends on the fluid properties (i.e. on the parameters $\xi, \zeta$ ); it is described in the Appendix A. The temperatures in the solid and the liquid differ and the temperature distributions deviate from linear. Their temperature difference remains however small because the wall heat exchange with the liquid is much stronger than with the gas.

The stationary solution is absent for evaporator power smaller than a threshold value, cf. Appendix A. This occurs because the

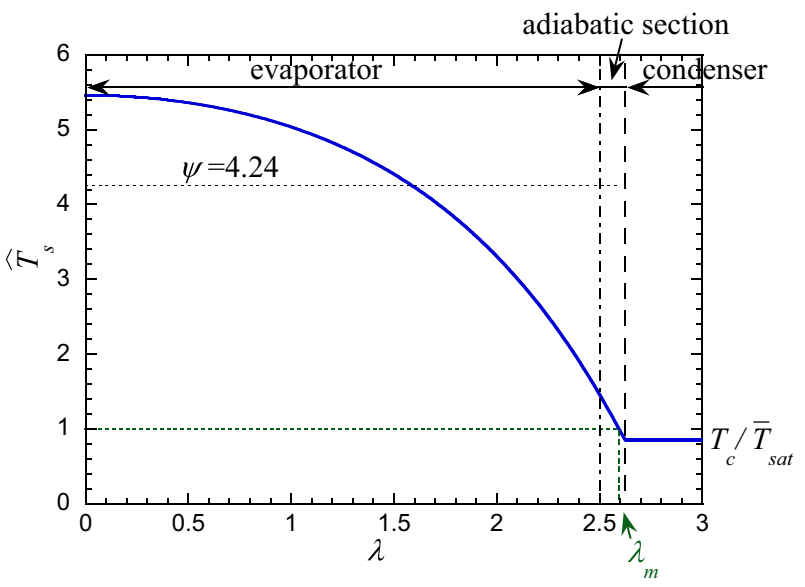

Fig. 2. Equilibrium temperature distribution along the PHP tube computed for $\beta=2, \alpha=2.5, \varepsilon=0.05$, and $\eta=0.15$ and result in $\chi=1.04$ (case A) and $\psi=4.24$ tube temperature at the meniscus location is imposed; it is the saturation temperature, cf. Eq. (19). To provide oscillations [7], it should be larger than $T_{c}$. Therefore, the heating power should be large enough to create high enough evaporator temperature for the continuity of heat flux along the tube at the meniscus location. Note that $\chi$ is determined from Eq. (A.5) which contains a group $\eta /\left(\beta \alpha^{2}\right)$ that depends on the evaporator power. For example, for $\varepsilon=0.15, \xi=100$ and $\zeta=700$, the stationary state is absent if $\eta /\left(\beta \alpha^{2}\right) \gtrsim 0.55$.

The stationary state existence threshold is defined for the dimensionless group $\eta /\left(\beta \alpha^{2}\right)$. This means that for fixed $P_{e}$, the stationary state is absent for $T_{c}$ smaller than some value defined by the above threshold or, for fixed $P_{e}$ and $T_{c}$, for $L_{e}$ smaller than some value.

It is evident that if the heating power is insufficient, the gas recondenses until the meniscus disappears. The oscillation cannot thus exist in such a case. As such a situation is not of interest, the recondensation regime boundary is not discussed any more.

\subsection{Temperature derivative at the equilibrium meniscus location}

It is a priori evident that the parameter $\hat{T}_{s}^{\prime}\left(\lambda_{m}\right)$ mentioned in the title of this section is an important quantity for the oscillation start-up. Indeed, during a small deviation from the equilibrium position, the meniscus should evolve in the temperature gradient given by this quantity. It is given by the expression

$\hat{T}_{s}^{\prime}\left(\lambda_{m}\right)= \begin{cases}-\alpha \beta, & \text { if } \alpha^{2} \beta \varepsilon \geqslant \eta, \\ -\alpha \beta \chi, & \text { otherwise. }\end{cases}$

The first of the options corresponding to the case A may be obtained from the last line of Eq. (29) and Eq. (30e); the second corresponds to case B and is equivalent to Eq. (A.4).

Such a result is somewhat counter-intuitive because one may imagine that the temperature gradient is defined by the difference between the condenser and evaporator temperatures divided by the sum of the evaporator and adiabatic lengths. However, the gradient in the most common case A depends neither on the condenser temperature nor on the adiabatic and evaporator lengths. It is defined only by the evaporator power.

\section{Stability analysis}

\subsection{Linearization}

To study the system stability, one needs to consider small deviations (denoted by $\Delta$ ) from the respective equilibrium values, e.g. $p=\bar{p}+\Delta p$. Then such expansions are substituted into the governing equations and only linear with respect to deviations terms are kept. Like previously [7], the resulting equations will not be linear but piece-wise linear (PWL). In what follows the equations written for deviations are presented in the dimensionless formulation; a dimensionless deviation is denoted with a tilde unless a special notation (like $\lambda$ for the dimensionless length) is introduced. The employed characteristic time and mass scales are, respectively,

$t_{s}=\frac{\rho_{s} c_{s} S_{s}}{\pi d U_{g}}$,

$m_{1}=\frac{\pi d U_{m} L_{m} \bar{T}_{s a t}}{h_{l g}} t_{s}$

while the pressure and power scales are $p_{r}$ and $p_{r} S L / t_{s}$, respectively. The time $t_{s}$ may be easily obtained by balancing the tube thermal inertia and the heat exchange of the gas with dry tube walls and thus characterizes the dynamics of the tube temperature variation. 
The following additional dimensionless constants appear in the dynamical problem:

$\sigma=\left.\frac{\partial T}{\partial p}\right|_{\text {sat }} \frac{p_{r}}{\bar{T}_{\text {sat }}}$,

$\theta=\frac{\pi d U_{g} \bar{T}_{s a t}}{p_{r} S} t_{s}$

$\mu=\alpha \chi \frac{m_{1}}{\bar{m}}=\theta \frac{U_{m}}{U_{g}} \frac{L_{m} R \bar{T}_{\text {sat }} \psi}{L h_{l g}}$,

$f=8 \pi v t_{s} / S$,

$\phi=\frac{D_{s}}{D_{l}}$,

$\gamma=1+R / c_{V}$

The most important feature of the analysis concerns Eq. (12). The linearization of the integral results in $\left(\Delta x_{\text {sat }}-\Delta x_{f}\right)\left[\bar{T}_{s}\left(\bar{x}_{\text {sat }}\right)-\bar{T}_{\text {sat }}\right]$, which vanishes because of the conditions $(18,19)$. This means that the film evaporation-condensation does not contribute to the linearized equations. Therefore, the film parameters like $\delta$ do not impact the stability conditions and the oscillation development is entirely controlled by the meniscus (and not the film) evaporation-condensation:

$\dot{\tilde{m}}_{f, e}=0$,

$\Delta \dot{\tilde{m}}=\Delta \dot{\tilde{m}}_{m}=\Delta \tilde{T}_{s}\left(\lambda_{\text {sat }}\right)-\Delta \tilde{T}_{\text {sat }}$.

In the last expression, one needs to account for the expansion $\lambda_{\text {sat }}=\lambda_{m}+\Delta \lambda_{m}$ :

$\Delta \widetilde{T}_{s}\left(\lambda_{\text {sat }}\right)=\Delta \tilde{T}_{s}\left(\lambda_{m}\right)+\hat{T}_{s}^{\prime}\left(\lambda_{m}\right) \Delta \lambda_{m}$

$\Delta \tilde{T}_{\text {sat }}=\sigma \Delta \tilde{p}$

that involves the $T_{S}$ derivative at the equilibrium meniscus position (33). The pressure variation $\Delta \tilde{p}$ is determined from Eq. (13),

$\Delta \tilde{p}=\frac{\mu}{\lambda_{m}} \Delta \tilde{m}+\frac{\Delta \tilde{T}}{\psi}-\frac{\Delta \lambda_{m}}{\lambda_{m}}$.

The remaining equations are

$\Delta \dot{\lambda}_{m}=\tilde{V}$

$\Delta \dot{\lambda}_{f}= \begin{cases}\tilde{V} & \text { if } \Delta \lambda_{f} \geqslant \Delta \lambda_{m} \text { and } \tilde{V}<0, \\ 0 & \text { otherwise, }\end{cases}$

$\frac{\lambda_{m}}{\psi(\gamma-1)} \Delta \dot{\tilde{T}}=\mu \Delta \dot{\tilde{m}}-\tilde{V}+\theta\left\{\int_{0}^{\lambda_{m}}\left[\Delta \tilde{T}_{s}(\lambda)-\Delta \tilde{T}\right] \mathrm{d} \lambda+\Delta \lambda_{f}(1-\psi)\right\}$

$\gamma \dot{\widetilde{V}}=\lambda_{m} \Omega^{2} \Delta \tilde{p}-\gamma f \widetilde{V}$

where the dimensionless eigenfrequency is $\Omega=t_{s} / t_{g}$, the characteristic time

$t_{g}=\sqrt{\frac{\rho_{l} \bar{x}_{s a t}\left(L_{t}-\bar{x}_{\text {sat }}\right)}{p_{r} \gamma}}$

being related to the oscillation period [6] via $\Pi=2 \pi t_{g}$. There are five ordinary differential Eqs. (38), (41)-(44) to be solved. Eq. (42) makes the problem piece-wise linear.

To linearize the heat diffusion problem, one neglects the nonlinearity introduced by the moving boundary (i.e. the meniscus) and considers the problems with two fixed intervals $\left[0, \lambda_{m}\right]$ and $\left[\lambda_{m}, \alpha(1+\varepsilon)\right]$

$\Delta \dot{\widetilde{T}}_{s}=\Delta \tilde{T}_{s}^{\prime \prime}+\left\{\begin{array}{ll}\Delta \tilde{T}-\Delta \tilde{T}_{s}, & \text { if } \lambda \in\left[0, \lambda_{m}\right] \\ \xi\left(\Delta \tilde{T}_{l}-\Delta \tilde{T}_{s}\right), & \text { if } \lambda \in\left[\lambda_{m}, \alpha(1+\varepsilon)\right]\end{array}\right.$,

$\phi \Delta \dot{\widetilde{T}}_{l}=\Delta \tilde{T}_{l}^{\prime \prime}+\xi \zeta\left(\Delta \tilde{T}_{s}-\Delta \tilde{T}_{l}\right)$,

with the boundary conditions
$\Delta \tilde{T}_{s}^{\prime}(0)=0, \quad \Delta \tilde{T}_{s}(\alpha(1+\varepsilon))=0$,

$\Delta \tilde{T}_{l}\left(\lambda_{m}\right)=\Delta \tilde{T}_{\text {sat }}, \quad \Delta \tilde{T}_{l}(\alpha(1+\varepsilon))=0$.

The chosen initial conditions are

$\widetilde{V}=\widetilde{V}_{0}, \quad \Delta \lambda_{f}=\Delta \tilde{m}=\Delta \widetilde{T}=\Delta \lambda_{m}=\Delta \widetilde{T}_{s}(\lambda)=\Delta \widetilde{T}_{l}(\lambda)=0$.

In what follows, we use the numerical value $\widetilde{V}_{0}=0.01 \Omega$.

The temporal evolution of the system can now be simulated. The right hand sides of the partial differential Eqs. (46) and (47) are discretized with the finite volume numerical method similarly to [4], cf. Fig. 3. There are $N_{g}+11 \mathrm{D}$ volume elements within the part of the solid $\left[0, \lambda_{m}\right]$ in contact with the gas, $N_{s}-N_{g}$ elements within the part $\left[\lambda_{m}, \alpha(1+\varepsilon)\right]$ in contact with the liquid, and the remaining elements in the liquid to result in $N_{t}$ total number of nodes. The lengths of the inner elements in two above regions are $2 e_{g}$ and $2 e_{l}$, respectively. The border elements are half-length. Their temperatures are given by three boundary conditions (48) so that there are $N_{t}-3$ unknown liquid and solid temperatures and the same number of the differential equations (written for each of the $N_{t}-3$ volume elements) that have become ordinary: the time derivatives are kept. The system of ordinary differential equations completed by five Eqs. (38), (41)-(44) is solved by the fourth order Runge-Kutta method using the initial conditions (50). A strong difference between $e_{g}$ and $e_{l}$ (in practice $e_{g}=10 e_{l}$ ) is required to resolve a much steeper temperature variation in the liquid domain. For numerical stability of the algorithm, the time step should be reduced for $\alpha<1$. Both the grid independence and convergence of such an algorithm have been checked.

For some parameters, the initial velocity fluctuation declines, for some grows with time. The temporal evolution of $\Delta \tilde{m}, \Delta \widetilde{T}, \Delta \lambda_{m}, \Delta \lambda_{f}$ is presented in Fig. 4a for the unstable case where the initial fluctuation develops. The evolution is similar to the fixed temperature case [7]. The nonlinearity of the system manifests itself through the nonzero quantities averaged over an oscillation period. While the meniscus always oscillates around the initial equilibrium position, the average gas temperature grows while the average gas mass decreases. This occurs because of the asymmetrical film edge dynamics. The film is left behind the receding liquid meniscus (Fig. 5a) and is "eaten up" when the meniscus advances (Fig. 5b). As a consequence, the gas is in contact with the hot portion of the tube but not with the cold. Due to the sensible heat exchange, the gas averaged temperature grows, its pressure grows too causing condensation and mass decrease.

The evolution of the solid and liquid temperature distributions along the tube during one oscillation period can be seen in Fig. 6 . Several particularities of the temperature distribution can be mentioned.

The tube temperature deviation $\Delta T_{s}$ (Fig. 6a) is constant along the major part of evaporator and only a thin boundary layer exists englobing the adiabatic section. This means that the tube temperature is controlled mainly by the gas-tube sensible heat exchange which is constant along the dry area. In (qualitative) agreement with the experimental data $[5,8]$, the tube temperature rises. This rise is monotonous because of large tube thermal inertia that smoothes out the heat exchange oscillations. The thermal

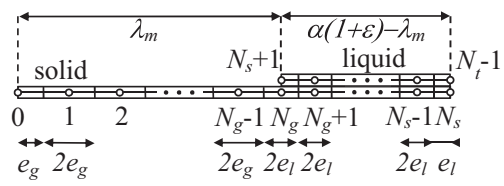

Fig. 3. The discretization scheme. The open circles are the node points in which the temperature is defined. The rectangles show the volume $1 \mathrm{D}$ elements. The liquid discretization copies that of the solid in the domain $\lambda>\lambda_{m}$. 


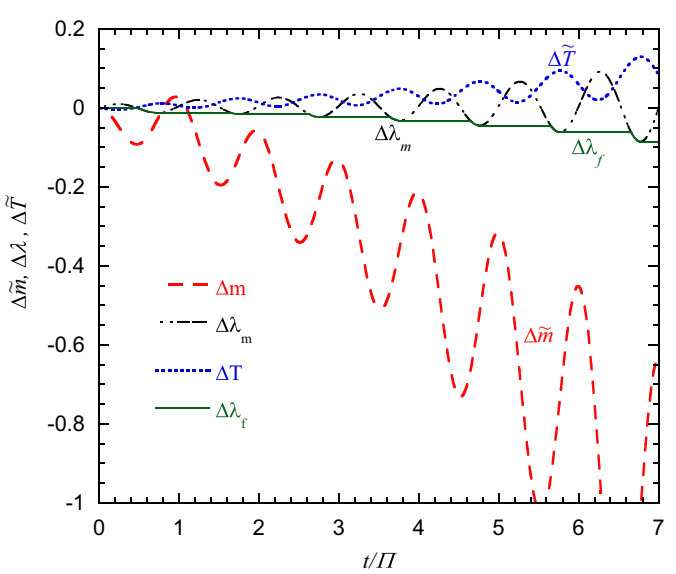

(a)

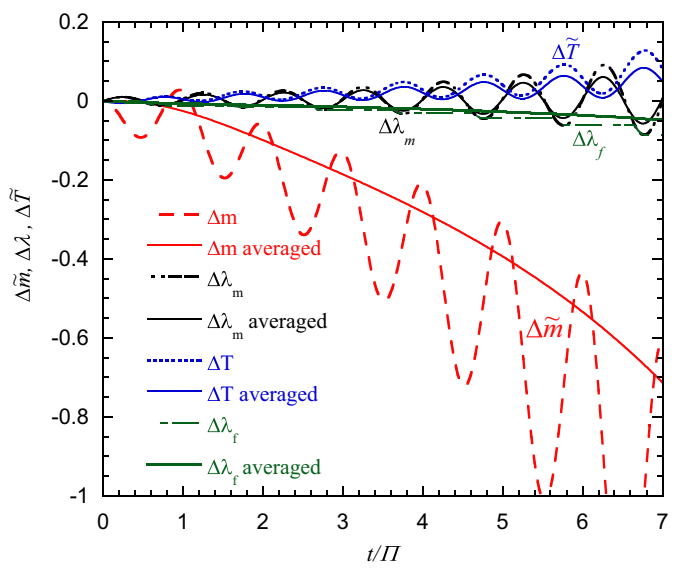

(b)

Fig. 4. Temporal evolution of system variables. The parameters $\gamma=1.36, \theta=160$, $\mu / \psi=6.4, \sigma=0.1, \Omega=800, \xi=100, \zeta=700, \phi=10^{3}$, and $f=3.2 \times 10^{-4}$ used hereafter are close to those of oxygen at $70 \mathrm{~K}[11]$. Other parameters are the same as for Fig. 2. The gas mass variation is rescaled: $\Delta \tilde{m}=\Delta m / m_{2}$. (a) Evolution accounting for the temporal temperature variation in the tube and in the liquid. (b) Comparison between the solution of the PWL set (51) and its averaging approximation (B.4) for the imposed temperature distribution.

boundary layer is caused mainly by the axial heat conduction toward the condenser. Its thickness grows slowly with time.

A boundary layer exists inside the liquid plug too (Fig. 6b). It is caused by the variation of the meniscus (saturation) temperature that follows the oscillating around $p_{r}$ gas pressure. The liquid boundary layer is much thinner than inside the solid.

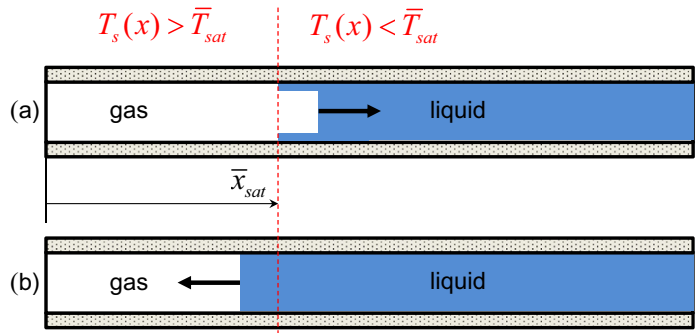

Fig. 5. Asymmetry of oscillations in the PHP during meniscus (a) receding and (b) advancing stages of oscillation.

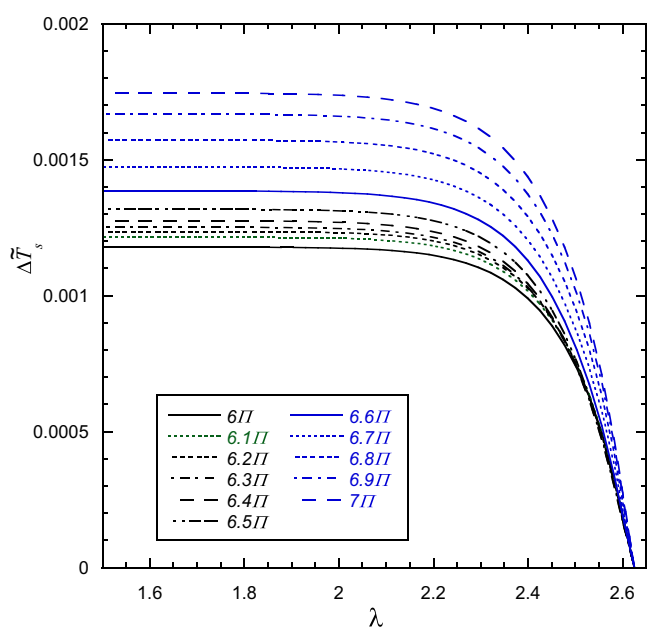

(a)

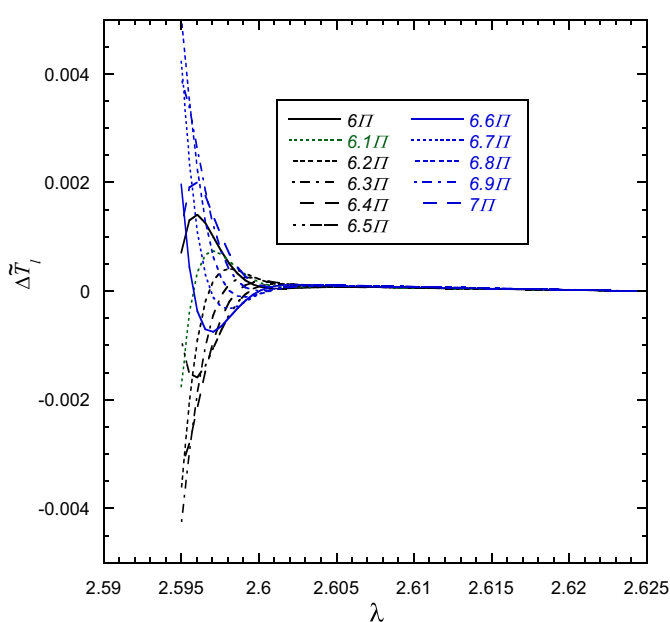

(b)

Fig. 6. Temporal evolution of the (a) tube and (b) liquid temperatures calculated for the same parameters as Figs. 2,4 for $6 \leqslant t / \Pi \leqslant 7$. The liquid domain corresponds to $\lambda>\lambda_{m} \simeq 2.595$.

\subsection{Simplified system: imposed $T_{s}(x)$ distribution}

One notices that the deviations of temperatures both of the tube and of the liquid are much smaller than the deviation of other temperatures (in particular, gas temperature), compare $\Delta \tilde{T}_{l, s} \sim 10^{-3}$ in Fig. 6 to $\Delta \tilde{T} \sim 0.1$ in Fig. 4a. It is thus reasonable to consider a simplified system with the imposed spatial variation of $T_{s, l}(x, t)=\bar{T}_{s, l}(x)$ constant in time. This corresponds to the case of a large thermal inertia of the tube. The solid-gas heat exchange is insufficiently strong to heat or cool the tube during an oscillation period. Since the solid-gas heat exchange is characterized by the time $t_{s}$ and the oscillations, by the time $t_{g}$, the large thermal inertia case corresponds to $\Omega=t_{s} / t_{g} \gg 1$. This case is often met in practice. Mathematically, one obtains the simplified equations by cancelling both $\Delta \tilde{T}_{s}=0$ from Eq. (43) and $\Delta \tilde{T}_{s}\left(\lambda_{m}\right)=0$ from Eq. (39). One mentions that the characteristic time $t_{s}$ becomes irrelevant. It is evident from the physical point of view. Indeed, as mentioned before, the time $t_{s}$ characterizes the tube cooling rate which is irrelevant within the imposed $T_{s}(x)$ model. Let us thus rescale the time with $t_{g}$. The rescaled equations read 
$\Delta \dot{\tilde{m}}=\hat{T}_{s}^{\prime}\left(\lambda_{m}\right) \Delta \lambda_{m}-\sigma\left(\frac{\check{\mu}}{\lambda_{m}} \Delta \tilde{m}+\frac{\Delta \tilde{T}}{\psi}-\frac{\Delta \lambda_{m}}{\lambda_{m}}\right)$,

$\frac{\lambda_{m}}{\psi(\gamma-1)} \Delta \dot{\widetilde{T}}=\check{\mu} \Delta \dot{\tilde{m}}-\tilde{V}+\check{\theta}\left[\Delta \lambda_{f}(1-\psi)-\lambda_{m} \Delta \tilde{T}\right]$

$\gamma \dot{\widetilde{V}}=\check{\mu} \Delta \tilde{m}+\frac{\lambda_{m}}{\psi} \Delta \tilde{T}-\Delta \lambda_{m}-\gamma \check{f} \widetilde{V}$

where the mass and velocity are now rescaled by the quantities $m_{2}=m_{1} / \Omega$, and $L / t_{g}$ independent of $t_{s}$. The rescaled constants $\check{\theta}=\theta / \Omega, \breve{\mu}=\mu / \Omega$, and $\check{f}=f / \Omega$ are independent of $t_{s}$ either. The other characteristic values and constants are not changed. Such a change corresponds to the replacement of $t_{s}$ by $t_{g}$ in Eqs. (35) and (36). Together with the expression (33), five ordinary differential Eqs. (41), (42), and (51) is a closed set to be solved with the initial conditions (50). The set is not linear (it is piecewise linear, PWL) and thus its stability cannot be studied with the classical linear stability analysis. Similarly to [7], the averaging method [14] can be applied. It consists in the identification of slowly varying (in comparison with the oscillation frequency) variables and the averaging over the remaining "fast" variables.

\subsection{Oscillation threshold}

The stability boundary (i.e. the oscillation threshold) can be obtained analytically with the averaging approximation as described in the Appendix B. It can be presented as a critical value of one of the parameters (e.g. heating power) as a function of all others. However we prefer the following formulation:

$-\hat{T}_{s}^{\prime}\left(\lambda_{m}\right)=\psi \frac{\theta}{\mu} \frac{(\gamma-1)^{2}}{\gamma}+\frac{\sigma \gamma}{\alpha \chi}+\frac{f}{\mu}$.

One recalls that the gradient of wall temperature at the equilibrium meniscus position $\hat{T}_{s}^{\prime}\left(\lambda_{m}\right)$ is negative (cf. Fig. 2). One can interpret Eq. (52) as a criterion expressing the critical value of $\left|\hat{T}_{s}^{\prime}\left(\lambda_{m}\right)\right|$ as a function of various parameters. The instability occurs if $\left|\hat{T}_{s}^{\prime}\left(\lambda_{m}\right)\right|$ is larger than this critical value. Such a criterion formulation is convenient because of the weak dependence of the r.h.s. of Eq. (52) on the system parameters. Indeed, the dependence on most parameters manifests itself through the parameters $\psi, \chi$. Both of them do not vary strongly. In particular, $\chi$ defines the equilibrium meniscus position: $\lambda_{m}=\alpha \chi$. Most often, this position is located close to the boundary between evaporator and adiabatic section (cf. Fig. 9 below) so that one can approximate $\chi \sim 1$.

The numerical solutions of all three considered above models (varying $T_{l, s}$, imposed $T_{l, s}$ and averaging approximation of the latter) can now be compared. One can see that the results of the varying (Fig. 4a) and imposed (Fig. 4b) $T_{l, s}$ models almost coincide. This is expected as $T_{l, s}$ variation (Fig. 6) is orders of value smaller than the variation of other temperatures, in particular gas temperature, cf. Fig. 4. Such a smallness is due to the choice of parameters corresponding to the large thermal inertia of both fluid and solid which is typical for PHPs. There is some discrepancy between the fixed $T_{l, s}$ PWL model and its averaging approximation, see Fig. $4 \mathrm{~b}$. Generally, the discrepancy decreases with the $\check{\theta}$ decrease, which is expected since $\check{\mu}$ is proportional to $\check{\theta}$ and the approximation becomes exact in the trivial limit $\check{\theta}, \check{\mu} \rightarrow 0$. The deviation increases with the $\mu / \theta$ decrease.

The stability boundary for the PWL equations may be found numerically by comparing the $\widetilde{V}$ oscillation amplitude after some time (the time $10 \Pi$ has been chosen) to its initial amplitude $\widetilde{V}_{0}$, the boundary corresponds to their equality. It should be mentioned that the boundary depends slightly upon this time; the boundary varies by about $1 \%$ when the time $20 \Pi$ is chosen. A comparison of the stability boundaries (as functions of $\alpha$, the other parameters being constant) obtained within three considered above models are shown in Fig. 7. The initial fluctuation declines below the respective curve and the oscillations develop above it.

One notices several features. (i) The PWL results of the imposed and varying temperatures virtually coincide. Such an agreement is expected because the tube thermal inertia is large due to $\Omega \gg 1$. Because of this, the imposed temperature PWL results will be discussed in the rest of the article. (ii) Although there is a $10 \%$ quantitative deviation of the averaging approximation from the PWL results, it reproduces well the qualitative tendency. Due to the analytical oscillation threshold expression (52), the approximation is thus useful to understand general tendencies. (iii) The successful power law fit of the averaged approximation boundary shows that the $\alpha$ dependence of the threshold value is provided mainly by the second term of Eq. (52). The $\alpha$ dependence of the threshold can thus be modeled by the term $\sigma \gamma / \alpha$.

Generally, a system becomes unstable when the energy input exceeds energy dissipation. There are two energy dissipation channels: the heat diffusion and the viscous losses. One can compare their contribution by comparing the last (viscous) term of Eq. (52) to the others. For the chosen parameters, the last term is of the order $10^{-5}$, while the others are of the order 1 . One concludes that the viscosity contribution is negligible unless the tube diameter is very small (because $f \sim S^{-1}$ ).

The dimensionless threshold for the temperature gradient (33) is defined for the only dimensionless group $\alpha \beta$ containing $k_{s}$ and $S_{s}$, which are the essential system parameters absent from other dimensionless groups. Indeed, the other dimensionless groups $\zeta$ and $\phi$ containing them has but a very small impact on the threshold (they impact only $\chi<1$ and $\Delta T_{l, s}$ ). Therefore a useful scaling law can be deduced: the start-up power scales like $\left(S_{S} k_{s}\right)^{1 / 2}$. This is expected since a larger heat conductivity requires a larger power to produce the same temperature gradient.

The instability boundary for different adiabatic section lengths is presented in Fig. 8.

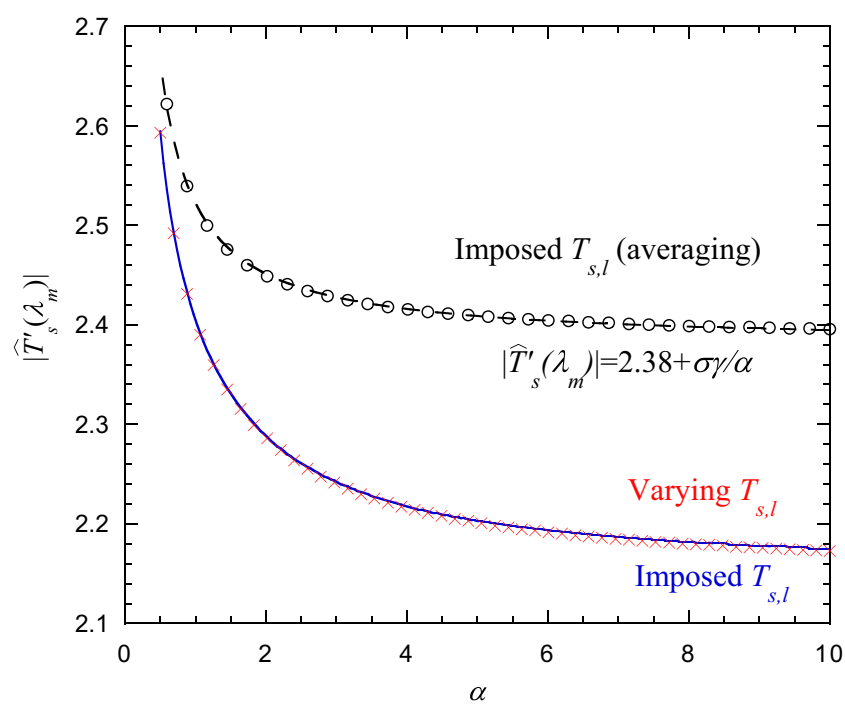

Fig. 7. Stability boundaries calculated for the same parameters as Figs. 2 and 4 . The boundaries are presented in terms of the axial temperature gradient $-\hat{T}_{s}^{\prime}\left(\lambda=\lambda_{m}\right)$ as a function of the dimensionless evaporator length $\alpha$. The boundaries calculated for the PWL models with the constant in time $T_{s, l}$ (solid line) and time varying $T_{s, l}$ (crosses) are compared to the averaging approximation of the imposed (constant in time) $T_{s, l}$ model (circles). The fit $-\hat{T}_{s}^{\prime}\left(\lambda_{m}\right)=2.38+\sigma \gamma / \alpha$ of the latter is shown with the dashed line. 


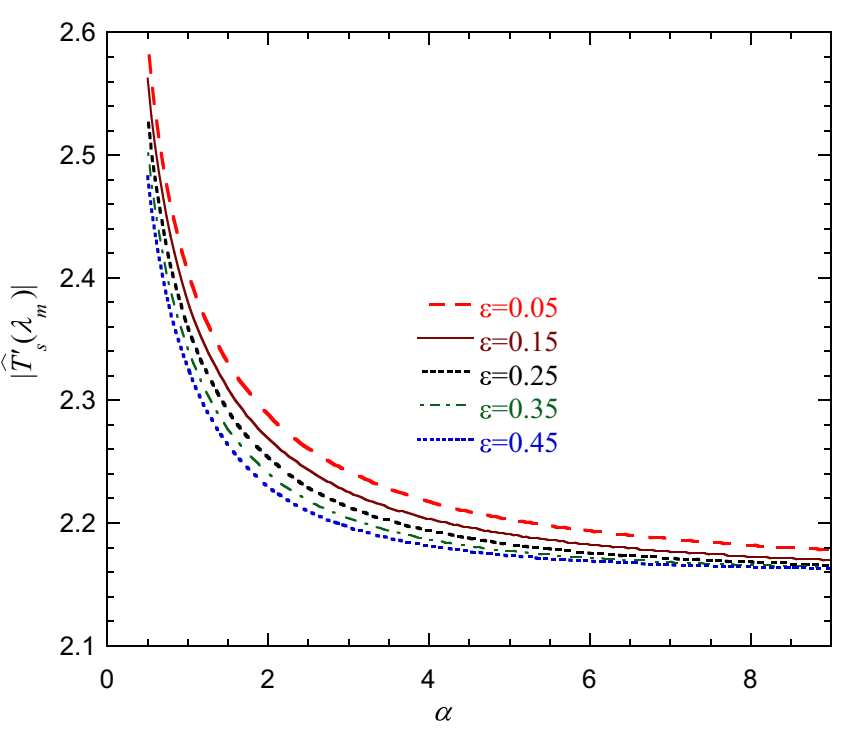

Fig. 8. The stability boundary calculated within the imposed $T_{s, l}$ PWL model in the coordinates $-\hat{T}_{s}^{\prime}\left(\lambda_{m}\right)$ and $\alpha$ for different dimensionless adiabatic section lengths $\varepsilon$. The other parameters are same as those used for Fig. 4.

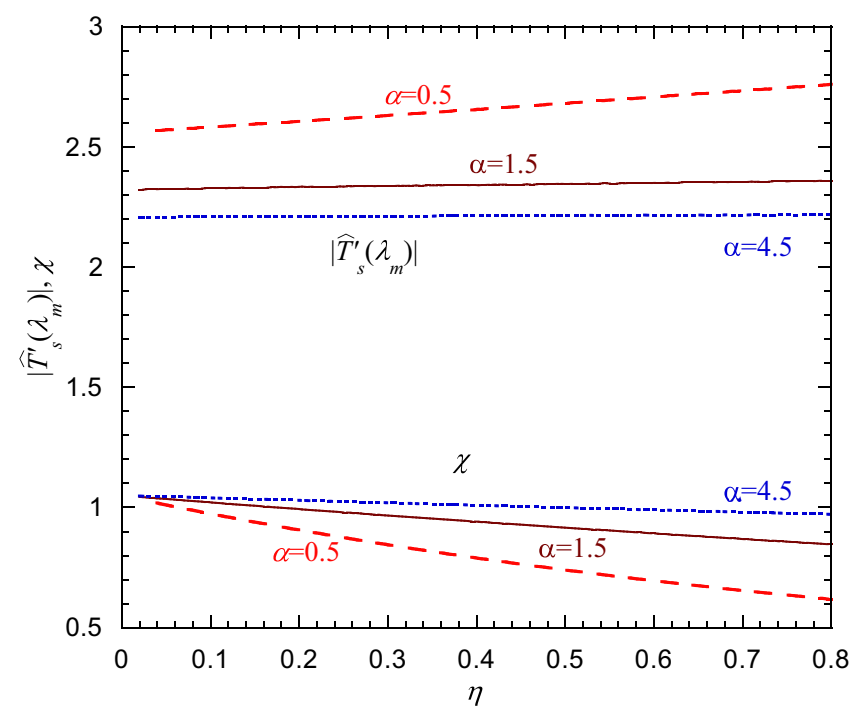

Fig. 9. The critical temperature gradient and the corresponding to it reduced meniscus position $\chi$ for the imposed $T_{s}$ model as functions of $\eta$ for different evaporator lengths $\alpha$ and $\varepsilon=0.05$. The other parameters are those of Fig. 8 .

The instability boundary dependence on the reduced condenser temperature $\eta$ is analyzed in Fig. 9 where the $\chi$ variation is also shown. One can see that the dependence of $\eta$ is quite weak. For large $\alpha$, this is explained by the independence of the temperature gradient (33) on $\eta$. Indeed, although $\eta$ increase causes $\chi$ decrease (cf. Eq. (30e)), the latter remains to be larger than unity, i.e. the meniscus remains to be inside the adiabatic section. The gradient remains invariable because the temperature profile is linear, see the last line of Eq. (29). For small $\alpha, \eta$ increase causes $\chi$ decrease beyond the unity where the gradient (33) is proportional to $\chi$. Finally the gradient value becomes to be insufficient any more for the oscillations start-up. Note that, although logical, such a behavior is counter-intuitive: one would expect a lower threshold for the lower $T_{c}$ (i.e. higher difference between evaporator and condenser temperatures). Such an effect appears due to the fact that our PHP is open so that the threshold is defined in terms of the difference of evaporator and saturation temperatures rather than evaporator and condenser temperatures. This feature is likely to be similar to the open multi-branch PHPs.

The numerical studies thus corroborate the result obtained analytically in the averaging approximation: the gradient critical value does not change much with other system parameters like adiabatic length, condenser temperature or liquid volatility (described by $\mu /(\psi \theta)$ ). One can see that the tube temperature gradient at the equilibrium meniscus position is indeed the right parameter to describe the stability threshold.

\section{Conclusions}

The oscillation inception in the single-branch PHP has been studied in the presence of the axial heat conduction in the PHP tube. A "soft" start-up regime caused rather by the meniscus/film evaporation than boiling has been considered.

First, the dynamic equilibrium system state has been found. At equilibrium, the liquid film is absent and the meniscus is located at a position where the tube temperature corresponds to the saturation temperature; the temperature distribution along the tube is nonlinear. The temporal evolution of the system responding to an initial fluctuation has been analyzed. The response is not linear even for small fluctuations because of asymmetry related to the liquid film deposition during the meniscus receding. During the initial fluctuation development, overall condensation occurs and the gas mass decreases. Both gas and tube temperature grow.

Next, the stability of the equilibrium state has been analyzed. The stability threshold corresponds to the start-up criterion. The main result of the above analysis is the independence of the start-up criterion of the liquid film properties. This result is very different from the previous model [7] and is a direct consequence of the heat conduction along the tube. Such a result means that the present analysis is quite universal since it is independent of a particular liquid film shape (flat, wedge-like, etc.).

The start-up criterion depends weakly on the liquid phase properties and quite strongly on the gas phase properties.

Among all the multitude of the PHP parameters, we have identified the most important parameter for which the start-up criterion should be defined. It is the temperature gradient along the tube, more specifically, its equilibrium value at the equilibrium meniscus location. It is related to the evaporator power and independent of the condenser temperature.

The present analysis shows a strong influence of the tube material and section on the PHP start-up. The start-up power scales like $\left(S_{s} k_{s}\right)^{1 / 2}$.

Since the above results have been obtained from the same mass transfer equations that apply to the multi-branch PHPs, the same conclusion should apply to them.

It has been found that when the tube thermal inertia is large (more specifically, when $\Omega \gg 1$ ), influence of the temporal variation of the tube temperature on the threshold can be neglected; only the equilibrium spatial temperature distribution along the tube matters. This is a common situation in experiments. In addition, such an assumption considerably simplifies the theoretical treatment.

Dependence of the start-up threshold on several system parameters has been analyzed. The threshold depends only weakly on condenser temperature or adiabatic section length. Such a situation is characteristic to the open PHP (that connected to a reservoir) for which the relevant parameter is the saturation temperature corresponding to the reservoir pressure rather than the condenser temperature. An analytical expression for the threshold has been obtained within the averaging approximation.

A PHP starts-up when the evaporator power exceeds energy dissipation. Two causes of energy dissipation has been considered: 
the heat dissipation by conduction (both in the solid and in the fluid) and liquid viscous dissipation. Usually the second dissipation channel is negligibly small with respect to the first. This tendency can however be inverse for tubes of microscopic diameters which should have higher start-up thresholds.

\section{Acknowledgements}

The financial contributions of ANR in the framework of the project AARDECO ANR-12-VPTT-005-02 and of ESA within MAP INWIP are acknowledged.

\section{Appendix A. Stationary state for the case of small evaporator power}

The case $\beta<\eta /\left(\alpha^{2} \varepsilon\right)$ (case B of Section 3.2) where $\chi<1$ will be considered here. Consider first the dry tube portion $0 \leqslant \lambda<\lambda_{m}$. The solution

$\hat{T}_{s}=\psi+\beta+C_{e} \cosh (\lambda)$

holds for $0 \leqslant \lambda<\lambda_{m}$ with the condition $\hat{T}_{s}\left(\lambda_{m}\right)=1$ that results in

$C_{e}=\frac{1-\psi-\beta}{\cosh (\alpha \chi)}$.

The $\psi$ value is found from the dimensionless counterpart of Eq. (24):

$\psi=1+\beta[\alpha \chi \operatorname{coth}(\alpha \chi)-1]$.

It is evident that $\psi>1$. By using Eq. (A.1), one obtains

$\hat{T}_{s}^{\prime}(\lambda-\alpha \chi \rightarrow-0)=-\alpha \beta \chi$.

Consider the conjugate problem (26), first for the interval $\alpha \leqslant \lambda \leqslant \alpha(1+\varepsilon)$. Let us use yet unknown $\hat{T}_{s, l}(\alpha)$ as given boundary conditions. Eq. (27) are used as two other boundary conditions. The solution of the linear set (26) on the above interval may now be found analytically with Wolfram Mathematica ${ }^{\circledR}$ software. The solution is straightforward but cumbersome and for this reason is not written here. For its checking, one may use the fact of the reduction to the linear function (cf. the last line of Eq. (29)) in the limit $\hat{T}_{s} \rightarrow \hat{T}_{l}$. From the obtained solution, one can now find the derivatives $\hat{T}_{s, l}^{\prime}(\alpha)$ as linear combinations of $\hat{T}_{s, l}(\alpha)$. The obtained pair of relations may be used as boundary conditions for the set (26) that needs to be solved now on the interval $\lambda_{m} \leqslant \lambda \leqslant \alpha$. The other pair of boundary conditions is $\hat{T}_{l, s}\left(\lambda_{m}\right)=1$. Mathematica can again be used to obtain analytically the solutions $\hat{T}_{l, s}(\lambda)$ which are now parameter-free. The variable $\chi$ remains to be the only unknown. It is found by matching the derivative $\hat{T}_{s}^{\prime}\left(\lambda-\lambda_{m} \rightarrow+0\right)$ with the value (A.4). Such a procedure guarantees that the obtained functions $\hat{T}_{l, s}(\lambda)$ are continuous and smooth.

The resulting equation $g(\chi) \equiv \hat{T}_{s}^{\prime}\left(\lambda-\lambda_{m} \rightarrow+0\right)+\alpha \beta \chi=0$ is nonlinear and needs to be solved numerically. Within a multiplier, it reads

$$
\begin{aligned}
& 2\left(e^{v \chi}-e^{v}\right)\left(e^{v \chi}-e^{v(2 \varepsilon+1)}\right)+v \frac{e^{2 v(\varepsilon+1)}-e^{2 v \chi}}{\varepsilon-\chi+1} \\
& \quad \times\left\{2 \zeta\left(\varepsilon-\frac{\eta}{\alpha^{2} \beta}\right)+2\left(\varepsilon \chi-\frac{\eta}{\alpha^{2} \beta}\right)+(1-\chi)[2 \chi+\zeta(\chi+1)]\right\}=0
\end{aligned}
$$

where $v=\alpha \sqrt{(\zeta+1) \xi}$. Fig. A.10 shows examples of $f(\chi)$ for different values of $\eta /\left(\alpha^{2} \beta\right)$ at constant $\varepsilon$. One can see that for large $\beta$ (small $\eta /\left(\alpha^{2} \beta\right)$ ) there is always a root corresponding to an equilibrium state. As required to have a cross-over between the cases $\mathrm{A}$ and $\mathrm{B}$, the root is $\chi=1$ when $\beta \alpha^{2} \varepsilon=\eta$. When $\beta$ decreases, the root value decreases. A second root may appear in a small range of parameters. The equilibrium states disappear when $\beta$ becomes inferior of a critical value.

\section{Appendix B. Application of the averaging method}

The consideration below is similar to the fixed temperature case described earlier [7].

Let us choose first a ground state that provides periodical oscillations. It can be defined by the equations

$\Delta \dot{\tilde{m}}=0$

$\Delta \dot{\lambda}_{m}=\widetilde{V}$

$\Delta \dot{\lambda}_{f}=0$,

$\frac{\lambda_{m}}{\psi} \Delta \dot{\widetilde{T}}=(1-\gamma) \widetilde{V}$,

$\gamma \dot{\widetilde{V}}=\frac{\lambda_{m}}{\psi} \Delta \widetilde{T}-\Delta \lambda_{m}+\check{\mu} \Delta \tilde{m}$

obtained from the set (41), (42), and (51) by omitting the terms responsible for the nonlinearity, mass transfer and energy dissipation (putting $\breve{f}=\breve{\mu}=\breve{\theta}=0$ ). The solutions of Eqs. (B.1) are indeed periodical with the unit eigenfrequency (cf. Fig. B.11),

$\widetilde{V}=r \sin \kappa$,

$\Delta \lambda_{m}=-r \cos \kappa+C$

$\Delta \widetilde{T}=\frac{\psi}{\lambda_{m}}[(\gamma-1) r \cos \kappa+E]$,

$\Delta \tilde{m}=(C-E) / \check{\mu}$,

where $\kappa=\tau+\varphi, \tau$ being the dimensionless time; for the moment, $r, \varphi, C, E$ are arbitrary constants. Eqs. (B.2) show that $\widetilde{V}, \lambda_{m}, \widetilde{T}$ are the "fast" variables (those that vary on the dimensional scale $t_{g}$ ). One may now reduce the initial set (51) with the method of variation of arbitrary constants. Assume now $r, \varphi, C, E, \Delta \lambda_{f}$ to be $\tau$ functions and substitute the expressions (B.2) back into Eqs. (41), (51). A straightforward reduction results in the equations

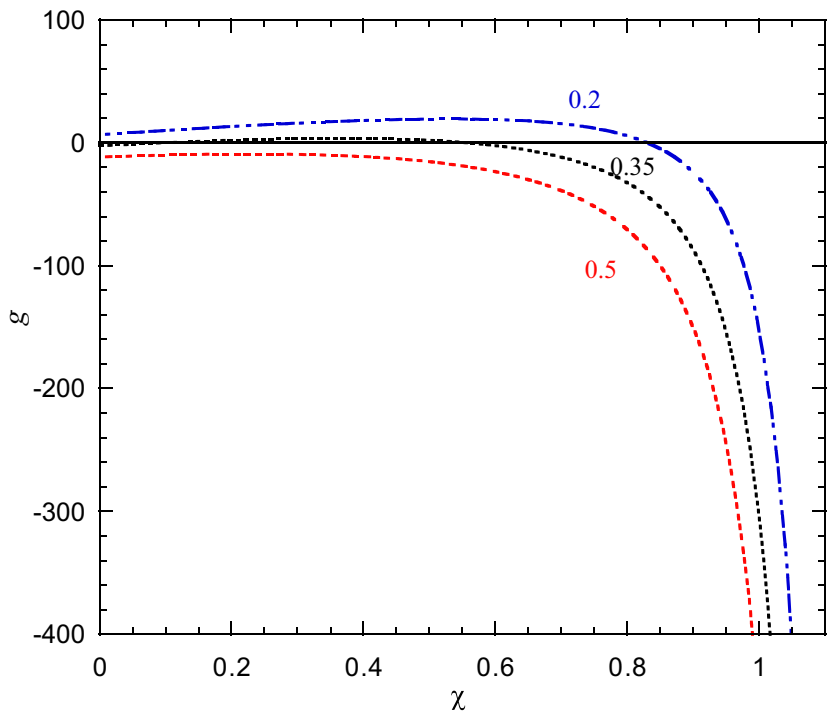

Fig. A.10. $g(\chi)$ function for different values of $\eta /\left(\alpha^{2} \beta\right)$ shown as a parameter for each curve. The other parameters $\zeta=1, \varepsilon=0.05, \alpha=0.5, \xi=10$ are fixed. 


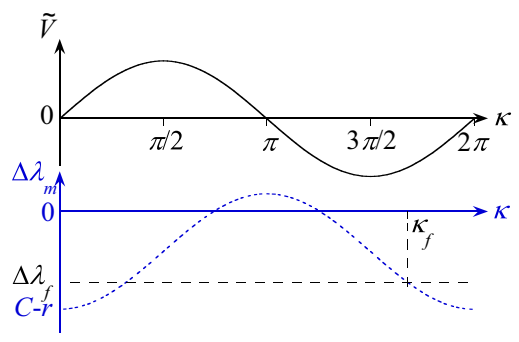

Fig. B.11. Ground state solutions (B.2a) (solid line) and (B.2b) (dotted line) of the nonlinear problem.

$\frac{1}{\check{\mu}}(\dot{C}-\dot{E})=\hat{T}_{s}^{\prime}\left(\lambda_{m}\right)(C-r \cos \kappa)-\frac{\sigma}{\lambda_{m}} \gamma r \cos \kappa$,

$\dot{r}=\dot{C} \cos \kappa-\check{f} r \sin ^{2} \kappa$,

$r \dot{\varphi}=-\dot{C} \sin \kappa-\check{f} r \sin \kappa \cos \kappa$,

$\frac{\gamma}{\check{\theta}(\gamma-1)} \dot{E}=\Delta \lambda_{f}(1-\psi)-\psi[(\gamma-1) r \cos \kappa+E]$.

which are still rigorous. The fifth equation is obtained by the trivial substitution of Eqs. (B.2a) and (B.2b) into Eq. (42), which results in a cumbersome expression omitted here. One may check by solving numerically these five equations together that all the variables $r, \varphi, C, E, \Delta \lambda_{f}$ exhibit small oscillations around slowly varying mean values and are thus the "slow" variables. Let us average Eqs. (42) and (B.3) over the oscillation period (i.e. integrate from 0 to $2 \pi$ and divide by $2 \pi$ ). While averaging the right hand side of the equations, $r, \varphi, C, E, \Delta \lambda_{f}$ (but not their derivatives) are to be assumed constant over the period. From now on, under $r, \varphi, C, E, \Delta \lambda_{f}$ we mean their averaged counterparts. One obtains from Eqs. (B.3):

$\frac{1}{\check{\mu}}(\dot{C}-\dot{E})=\hat{T}_{s}^{\prime}\left(\lambda_{m}\right) C$,

$\dot{r}=-\frac{r}{2}\left\{\check{\theta} \psi \frac{(\gamma-1)^{2}}{\gamma}+\check{\mu}\left[\hat{T}_{s}^{\prime}\left(\lambda_{m}\right)+\frac{\sigma \gamma}{\lambda_{m}}\right]+\check{f}\right\}$,

$\dot{E}=\check{\theta} \frac{\gamma-1}{\gamma}\left[(1-\psi) \Delta \lambda_{f}-\psi E\right]$,

and the condition $\dot{\varphi}=0$. Two cases are possible depending on the relation between $\Delta \lambda_{f}, C$ and $r$. Consider first the case where $\Delta \lambda_{f}$ is larger than the minimal value of $\Delta \lambda_{m}$ over the period (where the expression (B.2b) for $\Delta \lambda_{m}$ now uses the averaged values of $r, \varphi, C$ ), i.e. $\Delta \lambda_{f} \geqslant C-r$, cf. Fig. B.11. This case corresponds to a situation where the condition $\Delta \lambda_{f}=\Delta \lambda_{m}$ may be attained (i.e. the film disappears during some part of the period), which is justified both for the unstable regime and at the stability boundary. Indeed, since the film evaporation is neglected in the first-order approximation, the equality $\Delta \lambda_{f}=\Delta \lambda_{m}$ is attained when the amplitude grows in time or remains constant. For this case, Fig. B.11 shows that the averaging of Eq. (42) results in

$\Delta \dot{\lambda}_{f}=\frac{r}{2 \pi} \int_{\kappa_{f}}^{2 \pi} \sin \kappa d \kappa$

where $\kappa_{f}$ is defined by the condition $\cos \kappa_{f}=\left(C-\Delta \lambda_{f}\right) / r$ equivalent to $\Delta \lambda_{f}=\Delta \lambda_{m}$, cf. Eq. (B.2b).
The integration results in $\Delta \dot{\lambda}_{f}=-\frac{r}{2 \pi}\left(1-\cos \kappa_{f}\right)$. Finally,

$\Delta \dot{\lambda}_{f}=-\frac{1}{2 \pi}\left(r+\Delta \lambda_{f}-C\right)$

It is evident that the averaging of Eq. (42) for the case $\Delta \lambda_{f}<C-r$ (that means $\Delta \lambda_{f}<\Delta \lambda_{m}$ ) results in $\Delta \dot{\lambda}_{f}=0$.

To comply to the initial conditions (50), $r(0)=\widetilde{V}_{0}$, $C(0)=0, E(0)=0$, and $\varphi=\pi / 2$ need to be chosen. The set of Eqs. (B.4) is solved numerically. The solutions for $\Delta \lambda_{m}$ and other original variables is a result of substitution of the obtained functions $r(\tau), C(\tau), E(\tau)$ into Eqs. (B.2).

The stability threshold can be reasonably guessed without a rigorous analysis of the system (B.4) to be defined by the zero right hand side of Eq. (B.4b) (which results in the criterion (52)). Such a guess is based on the independence of this equation (the only one involving all the dissipation sources) from the others. The rigorous linear stability analysis done with the Hurwitz stability criterion (which is somewhat cumbersome and for this reason is not reproduced here) confirms this guess.

\section{References}

[1] S. Khandekar, P.K. Panigrahi, F. Lefèvre, J. Bonjour, Local hydrodynamics of flow in a pulsating heat pipe: a review, Front. Heat Pipes 1 (2) (2010) 023003, http://dx.doi.org/10.5098/fhp.v1.2.3003.

[2] V. Nikolayev, S.K. Sundararaj, Oscillating menisci and liquid films at evaporation/condensation, in: Proc. 17th Int. Heat Pipe Conf., IIT Kanpur, Kanpur, India, 2013.

[3] M.B. Shafii, A. Faghri, Y. Zhang, Thermal modeling of unlooped and looped pulsating heat pipes, J. Heat Transfer 123 (6) (2001) 1159-1172, http://dx.doi. org/10.1115/1.1409266.

[4] V.S. Nikolayev, A dynamic film model of the pulsating heat pipe, J. Heat Transfer 133 (8) (2011) 081504, http://dx.doi.org/10.1115/1.4003759.

[5] J. Qu, H. Wu, P. Cheng, Start-up, heat transfer and flow characteristics of silicon-based micro pulsating heat pipes, Int. J. Heat Mass Transfer 55 (21-22) (2012) 6109-6120, http://dx.doi.org/10.1016/j.ijheatmasstransfer.2012.06.024.

[6] S.P. Das, V.S. Nikolayev, F. Lefèvre, B. Pottier, S. Khandekar, J. Bonjour, Thermally induced two-phase oscillating flow inside a capillary tube, Int. J. Heat Mass Transfer 53 (19-20) (2010) 3905-3913, http://dx.doi.org/10.1016/j. ijheatmasstransfer.2010.05.009.

[7] V.S. Nikolayev, Oscillatory instability of the gas-liquid meniscus in a capillary under the imposed temperature difference, Int. J. Heat Mass Transfer 64 (2013) 313-321, http://dx.doi.org/10.1016/j.ijheatmasstransfer.2013.04.043.

[8] X. Liu, Y. Chen, M. Shi, Dynamic performance analysis on start-up of closedloop pulsating heat pipes (CLPHPs), Int. J. Therm. Sci. 65 (2013) 224-233, http://dx.doi.org/10.1016/j.ijthermalsci.2012.10.012.

[9] M. Rao, F. Lefèvre, S. Khandekar, J. Bonjour, Heat and mass transfer mechanisms of a self-sustained thermally driven oscillating liquid-vapour meniscus, Int. J. Heat Mass Transfer 86 (2015) 519-530, http://dx.doi.org/ 10.1016/j.ijheatmasstransfer.2015.03.015.

[10] B. Holley, A. Faghri, Analysis of pulsating heat pipe with capillary wick and varying channel diameter, Int. J. Heat Mass Transfer 48 (13) (2005) 26352651, http://dx.doi.org/10.1016/j.ijheatmasstransfer.2005.01.013.

[11] P. Gully, F. Bonnet, V. Nikolayev, N. Luchier, T.Q. Tran, Evaluation of the vapor thermodynamic state in PHP, in: Proc. 17th Int. Heat Pipe Conf., IIT Kanpur, Kanpur, India, 2013.

[12] V. Srinivasan, V. Marty-Jourjon, S. Khandekar, F. Lefèvre, J. Bonjour, Evaporation of an isolated liquid plug moving inside a capillary tube, Int. J. Heat Mass Transfer 89 (2015) 176-185, http://dx.doi.org/10.1016/j. ijheatmasstransfer.2015.05.039.

[13] V.S. Nikolayev, Comment on Flow and heat transfer of liquid plug and neighboring vapor slugs in a pulsating heat pipe by Yuan, Qu, \& Ma, Int. J. Heat Mass Transfer 54 (9-10) (2011) 2226-2227, http://dx.doi.org/10.1016/j. ijheatmasstransfer.2011.01.007.

[14] A. Nayfeh, Introduction to Perturbation Techniques, Wiley VCH, Weinheim, 2004, ISBN 9783527618453. 